\begin{tabular}{|c|l|}
\hline Title & $\begin{array}{l}\text { Dynamical Switching of a Reaction Coordinate to Carry the System through to a Different Product State at High } \\
\text { Energies }\end{array}$ \\
\hline Author(s) & Teramoto, Hiroshi; Toda, Mikito; Komatsuzaki, Tamiki \\
\hline Citation & $\begin{array}{l}\text { Physical Review Letters, 106(5), 054101 } \\
\text { https:/doi.org/10.1103/PhysRevLett.106.054101 }\end{array}$ \\
\hline Issue Date & 2011-02-04 \\
\hline Doc URL & http://hdl.handle.net/2115/44959 \\
\hline Rights & O2011 A merican Physical Society \\
\hline Type & article \\
\hline File Information & PRL106-5_054101.pdf \\
\hline
\end{tabular}

Instructions for use 


\title{
Dynamical Switching of a Reaction Coordinate to Carry the System through to a Different Product State at High Energies
}

\author{
Hiroshi Teramoto, ${ }^{1, *}$ Mikito Toda, ${ }^{2}$ and Tamiki Komatsuzaki ${ }^{1}$ \\ ${ }^{1}$ Molecule \& Life Nonlinear Sciences Laboratory, Research Institute for Electronic Science, Hokkaido University, \\ Kita 20 Nishi 10, Kita-ku, Sapporo 001-0020, Japan \\ ${ }^{2}$ Complex System Laboratory, Department of Physics, Faculty of Science, Nara Women's University, Nara, 630-8506, Japan
}

(Received 30 November 2010; published 31 January 2011)

\begin{abstract}
Questions of how the nature of a reaction coordinate that dominates the reaction ceases to exist and whether some new features emerge as an increase of total energy of systems are investigated for many degrees of freedom Hamiltonian systems. As a model system, a hydrogen atom in crossed electric and magnetic fields is scrutinized. It is shown that, when the total energy increases, the reaction coordinate no longer dominates the reaction as did at the lower energies. In turn, a new reaction coordinate emerges, connecting totally different reactant and product states. Furthermore, depending on which parts of the phase space the system traverses through the saddle, the system nonuniformly experiences the switching of the reaction coordinate leading to the different product state. The universal mechanism of the cessation and the switching of the reaction coordinate at high energy regimes above the saddle is investigated.
\end{abstract}

The mechanism of how a system changes its state is one of the most fundamental subjects in a variety of fields such as not just chemistry [1-3], but also celestial mechanics [4-6], fluid mechanics [7,8], environmental science [9] and biology [10]. For instance, the question of how an asteroid transports from a planet to another under the influence of gravitational forces, or how the route of a spacecraft for the space mission can be designed so as to minimize the consumption of the fuel is one of the fundamental issues in celestial mechanics [6]. Recent developments in the theory of reaction dynamics have succeeded in bridging such apparently different transport phenomena on the common basis of the geometrical structure of the underlying phase space in many degrees of freedom (DOF) systems [11].

The central building blocks in the phase space for the understanding of those transport phenomena are "normally hyperbolic invariant manifolds (NHIMs)" and the stable and unstable manifolds emanating along the normal direction to the NHIM. Here, NHIM is an invariant manifold such that the instability (stability) normal to that manifold is more prominent than that tangential to it, which is called normal hyperbolicity [12]. For example, the way of how the stable and unstable manifolds intersect with each other governs the global nature of the transport both in Hamiltonian [13] and dissipative [14] systems. In the context of reaction dynamics, the normal direction to the NHIM serves as a (phase space) reaction coordinate that dominates the reaction, which is locally decoupled from the other coordinates even under the existence of chaos [1-3].

NHIM is proven to be structurally stable as long as it is compact and normally hyperbolic [12], but only a few studies have addressed the loss of those properties of
NHIM and its consequences [15-17], such as nondefinability of no-return transition state [15] and universal scaling laws appearing on the verge of the breakdown of NHIM [16]. Yet the question of what emerges after the breakdown of NHIM has been still one of the most intriguing problems to be uncovered. Since the normal hyperbolicity of NHIM ensures the existence of the phase space coordinate to dominate the reaction, the problem of the breakdown of NHIM and its consequences are directly related to the question: "How does the reaction coordinate cease to exist and what emerges after the cessation?"

In this Letter, we look into the mechanism of why and how the nature of the reaction coordinate that dominates all reactions becomes ruined as the total energy of the system increases far above the energy regime where the NHIM persists. It is found, in a model system of a hydrogen atom in crossed electric and magnetic fields, that the system dynamically experiences a switching of the reaction coordinate connecting totally different reactant and product states at the high energy regime. The universal feature for many-DOF systems is investigated by the analyses of local instability of the dynamics along unstable periodic orbits buried in the NHIM.

The Hamiltonian for a hydrogen atom in crossed electric and magnetic fields is expressed by

$$
H=\frac{1}{2}\left(P_{1}-\frac{x_{2}}{2}\right)^{2}+\frac{1}{2}\left(P_{2}+\frac{x_{1}}{2}\right)^{2}+\frac{1}{2} P_{3}^{2}-\frac{1}{R}-\epsilon x_{1},
$$

where $x_{i}, P_{i}(i=1,2,3)$ are the $i$ th (scaled) Cartesian coordinate and its conjugate momentum, $R=$ $\sqrt{\left(x_{1}-x_{s}\right)^{2}+x_{2}^{2}+x_{3}^{2}}, \quad x_{s}=-\epsilon^{1 / 2}$ and $\epsilon$, the (scaled) electric field, is the only remaining parameter after the scaling of the original variables and energy in atomic 
units [2]. This system has a saddle point at the origin which is called the Stark saddle that connects two distinct states: one in which the electron is bound to the nuclei and the other in which it is not. This saddle is of rank one, that is, it has only one unstable mode along which to leave to either of the states while all the other modes are stable. Followed by a coordinate transformation, the quadratic part of the Hamiltonian around the origin can be diagonalized by a linear transformation [2]. The resulting Hamiltonian can be expressed by

$H(\xi, \eta, \mathbf{p}, \mathbf{q})=\lambda \xi \eta+\sum_{\ell=1}^{2} \frac{\omega_{\ell}}{2}\left(p_{\ell}^{2}+q_{\ell}^{2}\right)+\sum_{i, j \geq 0} c_{i j}(\mathbf{p}, \mathbf{q}) \xi^{i} \eta^{j}$,

where $\xi$ and $\eta$ denote the linear combinations of the normal coordinate and conjugate momentum of the reactive mode, which represent the stable and unstable directions around the saddle, and $p_{\ell}$ and $q_{\ell}$ the normal coordinates and the conjugate momenta of the nonreactive modes, respectively. In addition the terms $c_{i j}(\mathbf{p}, \mathbf{q}) \xi^{i} \eta^{j}$ start from the order of 3 with respect to $(\xi, \eta, \mathbf{p}, \mathbf{q}) . \lambda$ is a curvature along the unstable normal coordinate and $\omega_{1}, \omega_{2}$ are two frequencies of the nonreactive normal coordinates, which can be adjusted by $\epsilon$ [2]. Here, we chose $\epsilon$ so that $\omega_{1}, \omega_{2}$ satisfy 2:1 resonance, i.e. $\omega_{2} / \omega_{1}=$ $1 / 2$. If the energy of the system $E$ is sufficiently close to that of the saddle point, $(\xi, \eta)$ can be regarded as the reaction coordinate.

In order to elucidate the relative instabilities among the DOFs in the region of the saddle, we extract the NHIM, denoted by $M$ hereinafter, by transforming Eq. (2) to the following form [18] in the vicinity of the origin:

$\bar{H}(\bar{\xi}, \bar{\eta}, \overline{\mathbf{p}}, \overline{\mathbf{q}})=\lambda \bar{\xi} \bar{\eta}+\sum_{\ell=1}^{2} \frac{\omega_{\ell}}{2}\left(\bar{p}_{\ell}^{2}+\bar{q}_{\ell}^{2}\right)+\sum_{\substack{i+j \neq 1 \\ i, j \geq 0}} \bar{c}_{i j}(\overline{\mathbf{p}}, \overline{\mathbf{q}}) \bar{\xi}^{i} \bar{\eta}^{j}$.

Here, again, the order of $\bar{c}_{i j}(\overline{\mathbf{p}}, \overline{\mathbf{q}}) \bar{\xi}^{i} \bar{\eta}^{j}$ is not less than 3 with respect to $(\bar{\xi}, \bar{\eta}, \overline{\mathbf{p}}, \overline{\mathbf{q}})$. The $M$ is defined by the intersection between an equi-energy surface and a subset of phase space that satisfies $\bar{\xi}=\bar{\eta}=0$, which is an invariant set of Eq. (3) and the dynamics inside it is described by the reduced Hamiltonian $\bar{H}(0,0, \overline{\mathbf{p}}, \overline{\mathbf{q}})$.

To see how the dynamics in the $M$ qualitatively changes as $E$ increases, we show a Poincaré surface defined by $\bar{q}_{1}=0\left(\bar{p}_{1}>0\right)$ at two different energies in Fig. 1. The more $E$ increases, the more the dynamics in the $M$ becomes chaotic. This indicates that, as an increase of $E$, the instabilities of the dynamics grow in the tangential space of the $M$. Hence, it is expected that, when $E$ reaches a certain high energy, the developed chaos in the $M$ makes the instability of the dynamics greater than that of the normal direction to the $M$. This should not only spoil the $M$ but also give birth to new features in reactions because the
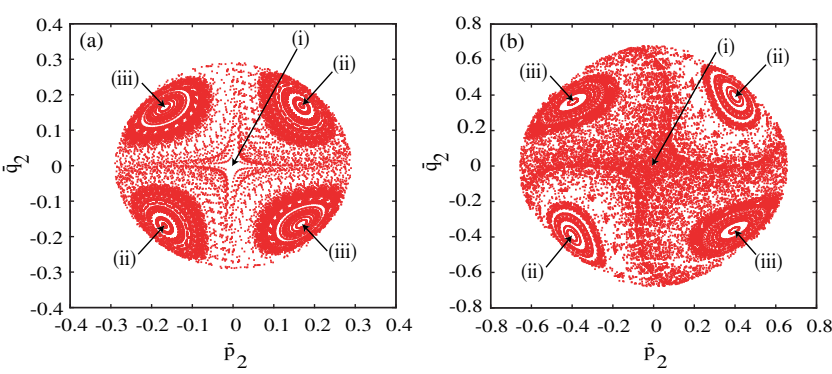

FIG. 1 (color online). The Poincaré surface of section $\left(\bar{p}_{2}, \bar{q}_{2}\right)$ of $\bar{q}_{1}=0$ with $\bar{p}_{1}>0$ obeying the reduced Hamiltonian $\bar{H}(0,0$, $\overline{\mathbf{p}}, \overline{\mathbf{q}})$. The reduced Hamiltonian was computed up to 18 th power in the original coordinates and momenta in Eq. (2) in the normal form calculation. (a) $E=0.02$. (b) $E=0.12$. (i)-(iii) denote an unstable fixed point (i) and stable period-two periodic points (ii), (iii).

nature of the original reaction coordinate at the low energy regime should also cease.

To explore the phase space structure at such a high energy regime, normal form theories are not so beneficial because normal form always suffers from a truncation error up to a certain finite order in the variables, which is usually based on a Taylor expansion of the Hamiltonian system in the vicinity of the saddle point. Furthermore, there is no guarantee that the normal form converges [18]. Therefore, it is vital to develop other techniques for this purpose.

The crux is to focus on unstable periodic orbits buried in the NHIM. For Hamiltonian systems, each periodic orbit exists as a continuous family with respect to the energy $E$ unless it bifurcates [19]. If a periodic orbit is in the NHIM at some energy regimes, its continuous family should also be in the NHIM unless the NHIM breaks down. It is because, in some open neighborhoods of the NHIM, none of the periodic orbits can exist outside of the NHIM due to the isolation property of NHIM [20]. Therefore, by following each bifurcation branch of periodic orbits in the NHIM as a function of $E$, we can trace the NHIM along the continuous family of the periodic orbits.

Figure 2 shows the bifurcation diagram of the periodic orbits in the $M$ originated from a set of normal modes at $E \simeq 0$, where the calculation was performed using the original Hamiltonian Eq. (1) and for the continuation of those periodic branches, we used Keller's pseudoarclength continuation [21]. There exist the other types of periodic orbits in the $M$, which are not shown in the figure; periodic orbits on a resonant torus, pairs of periodic orbits that originated from the resonant torus (Poincaré-Birkhoff theorem), periodic orbits generated by saddle-node bifurcation, $k$-bifurcation and Hamiltonian Hopf bifurcation [19].

In order to quantify the energy $(E)$ dependency of the ratio between the instability in the tangential direction and that in the normal direction, Fig. 2(b) presents the absolute values of the two characteristic multipliers, which are eigenvalues of the phase space Jacobian matrix 

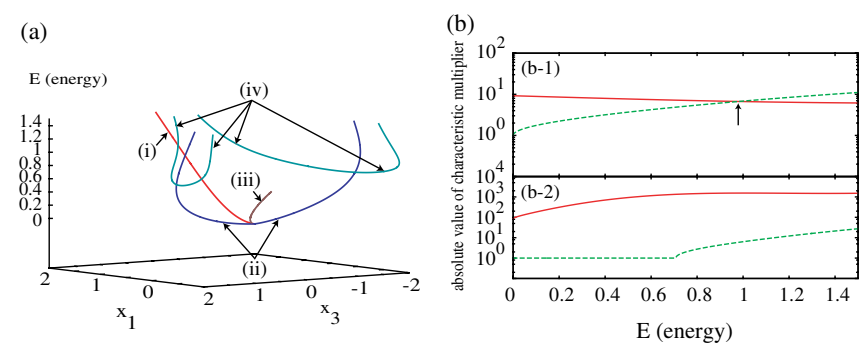

FIG. 2 (color online). (a) A bifurcation diagram of periodic orbits in the $M$ as a function of the total energy $E$ on the Poincare surface defined by $x_{2}=0, P_{2}>0$. These periodic orbits originated from the normal modes at the saddle point at $E \simeq 0$. Here (i), (ii), and (iii) correspond to that in Fig. 1, respectively. (i), (ii): periodic orbits that originated from the normal modes, (iii): a newborn periodic orbit bifurcated from (i) due to a perioddoubling bifurcation (the two periodic points degenerate here, which leave this Poincaré surface at $E=0.5130$ ), (iv): two pairs of periodic orbits bifurcated from each (ii) due to a pitchfork bifurcation. (b) The absolute values of the characteristic multiplier of the two unstable directions, that is, normal (thin line) and tangential (dotted line) directions, on the periodic orbit (i) (b-1) and (ii) (b-2) as a function of $E$.

(monodromy matrix) along a periodic orbit, for the periodic orbit (i) (b-1) which is located on the center of the strong chaotic region and for (ii) (b-2) which is located on the center of the torus region (c.f. Fig. 1). We identified the normal and tangential directions of the periodic orbits to the $M$ as follows: at slightly above the saddle point energy (i.e., $E \simeq 0$ ) we compute the normal form of Eq. (3) and identify the direction of normal and tangential directions to the $M$. We then evaluate the characteristic multipliers and the associated eigenvectors of the periodic orbit at the energy, identify each as either the normal or tangential direction and continue them along the family of each periodic orbit using Eq. (1). On the periodic orbit (i), normal hyperbolicity breaks down at $E \approx 0.99$ [indicated by the arrow in Fig. 2 (b-1)] whereas normal hyperbolicity survives on the periodic orbit (ii) up to $E=1.5$ [See Fig. 2 (b-2)].

Figure 3(a) shows a schematic portrait of the phase space around the periodic orbit (i) in the $M$ on the Poincaré surface $x_{2}=0, P_{2}>0$. On the Poincaré surface, the periodic orbit (i) becomes a fixed point [indicated by a red star in Fig. 3(a)] and it has two unstable directions: one is the direction $\left(u_{1}\right)$ normal to the $M$, the coordinate that originally dominates the reaction at energies slightly above the saddle point energy, and the other is the unstable direction $\left(u_{2}\right)$ tangential to the $M$. Thus the unstable manifold of the fixed point is a two-dimensional sheet on the Poincaré surface as drawn in Fig. 3(a), which is locally coordinated by these two unstable directions $\left(u_{1}, u_{2}\right)$. (The fixed point that corresponds to the periodic orbit (i) is set to the origin $\left(u_{1}, u_{2}\right)=\mathbf{0}$.)

To capture the change of the phase space structure as an increase of the energy $E$, around the periodic orbit (i),
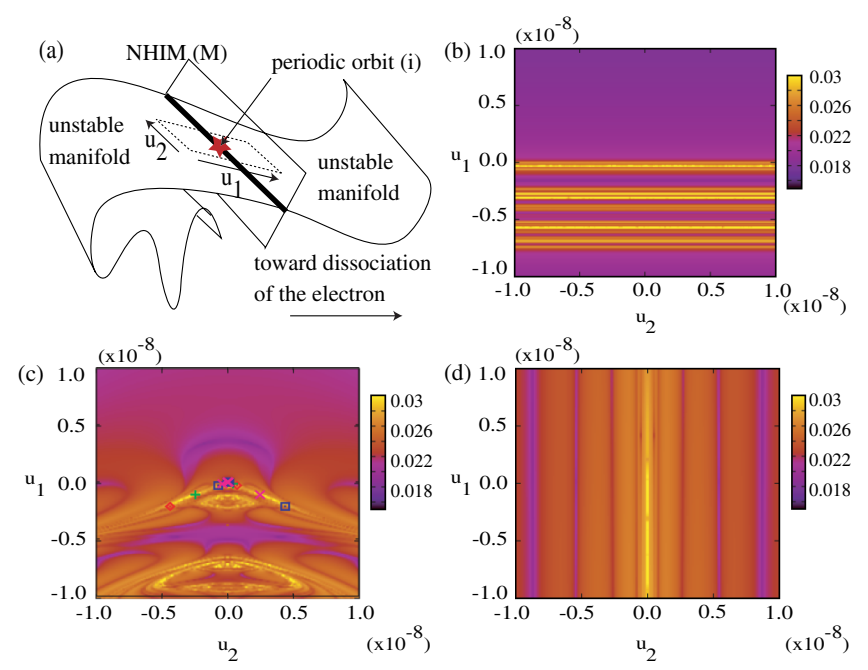

FIG. 3 (color online). (a) A phase space portrait around the periodic orbit (i) in the $M$ on the Poincaré surface $x_{2}=0$ and $P_{2}>0$. On the surface, the periodic orbit (i) becomes a fixed point (indicated by the star) and it has two unstable directions, $u_{1}$ and $u_{2}$. The unstable manifold of the fixed point is a two dimensional sheet, which is locally spanned by $u_{1}$ and $u_{2}$, and its intersection with the $M$ yields a one-dimensional curve, denoted by thick bold line. (b)-(d) The first local Lyapunov exponent distributions on the $u_{1}-u_{2}$ plane at three energies: (b) $E=0.08$, (c) $E=0.90$ [Some of the homoclinic orbits of the periodic orbit (i), which used to be in the $M$ at the low energy regime, are superposed (each symbol represents the corresponding homoclinic points).] and (d) $E=1.45$. The brighter (the more yellowish) the color, the larger the exponent. The exponents were computed for each trajectory sampled from the $u_{1}-u_{2}$ plane, with a time length of $1000 \approx 100 \times 2 \pi / \omega_{2}$.

we examine the first local Lyapunov exponent distributions on the $u_{1}-u_{2}$ plane [indicated by a dotted square in Fig. 3(a)] at three different energies in Fig. 3(b)-3(d). The existence and the location of repelling invariant objects can be identified as ridges of the first local Lyapunov exponent $[7,22,23]$. At just slightly above the saddle point energy, i.e., $E=0.08$ [Fig. 3(b)], a set of clear ridges of the first local Lyapunov exponent in parallel to the $u_{2}$ axis manifests the existence of the larger instability in the $u_{1}$ axis, implying that the $u_{1}$ serves as a dominant reaction coordinate. Here one ridge that runs through the origin corresponds to the intersection between the $M$ and the unstable manifold of the periodic orbit (i) because the origin is in the $M$ and the set of points emanating from the origin along the direction $\left(u_{2}\right)$ tangential to $M$ is also in the $M$. On the other hand, the other ridges correspond to the homoclinic-heteroclinic intersections of the unstable manifold of the periodic orbit (i) and the stable manifold of the $M$.

However, as the energy increases, approximately, $E=0.90$, the ridges become obscure and they no longer divide the space into two distinct parts. It was found that the part of the remaining ridges corresponds to the 
homoclinic orbits emanating from the periodic orbit (i) that used to be in the $M$ at the low energy regime, which are superposed on Fig. 3(c). These homoclinic orbits perform as a part of the remnant of the NHIM and persistently exist even after the NHIM breaks down as long as they correspond to the transversal intersections of the stable and unstable manifolds of the periodic orbit (i).

The most striking consequence at the high energy regime is this: as $E$ increases even higher, approximately, $E=1.45$ [See Fig. 3(d)], a set of new ridges that are parallel to the $u_{1}$ axis emerges. This implies that the $u_{1}$ axis, which used to be the reaction coordinate that dominated the reaction at the lower energies, starts to serve as a dividing surface, and, in turn, the $u_{2}$ axis starts to do as a new reaction coordinate to connect the different reactant and product states, which is regarded as the major reaction at the high energies [at least in the neighborhood of the periodic orbit (i)]. These new reactant and product states correspond to the phase space regions where the electron moves around the periodic orbit (ii) and some bifurcation branches of the periodic orbit (iii), respectively. The stable and unstable manifolds of the intersection between the invariant surface $\left(x_{3}, P_{3}\right)=(0,0)$ of the Hamiltonian (1) and the equi-energy surface provide the new reaction coordinate of the system that connects the new reactant and product.

The concept of reaction coordinate, in the context of chemical reaction theories, has been thought to be an object that only depends on the morphology of the underlying potential energy surface. Recent theoretical developments $[1,2]$ have revealed that the reaction coordinate is, just as postulated by Wigner [24], a dynamic entity that generally depends on the phase space structure in manyDOF systems. In this Letter, we presented a scenario of how the ruin of the reaction coordinate gives birth to its switching to a new reaction coordinate that connects different reactant and product states. It should be noted that along the other periodic orbits [(ii)-(iv)] which trace the other branches in Fig. 2, it was found that the original normal direction to the $M$ yet serves as the reaction coordinate, that is, the instability normal to the $M$ is yet larger than that tangential to it up to $E=1.50$ [e.g., See Fig. 2 (b-2)]. This implies that the dominant reaction coordinate changes depending on which regions in the phase space the system traverses through the saddle. These results provide new insights in revealing the mechanism of reactions far above the saddle energy where the NHIM no longer exists. Regarding possible manifestations for reaction kinetics, the breakdown of the NHIM would give rise to more chaotic, stochastic dynamics even in the vicinity of the saddle, which might yield Markovian reaction kinetics. On the other hand, the switching results in not just the alteration of the reaction rates but substantial structural changes in the reaction kinetic scheme.
This work has been supported by JSPS, Priority Area "Molecular Theory for Real Systems," by Grant-in-Aid for Young Scientists (B) from The Ministry of Education, Culture, Sports, Science and Technologyand by Research Center for Computational Science, Okazaki, Japan.

*teramoto@es.hokudai.ac.jp

[1] T. Komatsuzaki and R. S. Berry, Proc. Natl. Acad. Sci. U.S.A. 98, 7666 (2001).

[2] T. Uzer, C. Jaffe, J. Palacián, P. Yanguas, and S. Wiggins, Nonlinearity 15, 957 (2002).

[3] H. Waalkens, A. Burbanks, and S. Wiggins, J. Chem. Phys. 121, 6207 (2004).

[4] W.S. Koon, M. W. Lo, J.E. Marsden, and S.D. Ross, Contemp. Math. 292, 129 (2002).

[5] A. Celletti and L. Ferrara, Celest. Mech. Dyn. Astron. 64, 261 (1996).

[6] J.E. Marsden and S. D. Ross, Bull. Am. Math. Soc. 43, 43 (2005).

[7] G. Haller and A.C. Poje, Physica (Amsterdam) 119D , 352 (1998).

[8] G. Kawahara, Phys. Fluids 17, 041702 (2005).

[9] C. Coulliette, F. Lekien, J. Paduano, G. Haller, and J. Marsden, Environ. Sci. Technol. 41, 6562 (2007).

[10] B. Aldridge, G. Haller, P. Sorger, and D. Lauffenburger, IEE Proc. Syst. Bio. 153, 425 (2006).

[11] Geometric Structures of Phase Space in Multidimensional Chaos: Applications to Chemical Reaction Dynamics in Complex Systems, Adv. Chem. Phys. Vol. 130, edited by M. Toda, T. Komatsuzaki, T. Konishi, R. S. Berry, and S. A. Rice (John Wiley \& Sons, New York, 2005), Part A and B .

[12] N. Fenichel, Indiana University Mathematics Journal 21, 193 (1971).

[13] S. Wiggins, Chaotic Transport in Dynamical Systems (Springer, New York 1985), 4th ed..

[14] Y. Nishiura, Far-from-Equilibrium Dynamics, Translations of Mathematical Monograph Vol. 209 (American Mathematical Society, Providence, 2002).

[15] C. B. Li, A. Shoujiguchi, M. Toda, and T. Komatsuzaki, Phys. Rev. Lett. 97, 028302 (2006).

[16] A. Halo, Chaos 16, 013120 2006).

[17] D. G. Yang, arXiv0912.5419v1.

[18] C. Simó, CISM Course on Modern Methods of Analytical Mechanics and their Applications, Udine, June 16 to 20 (1997), http://www.maia.ub.es/dsg/1997/index.html

[19] K. R. Meyer, G. R. Hall, and D. Offin, Introduction to Hamiltonian Dynamical Systems and the N-Body Problem (Springer, New York, 2009), 2nd ed..

[20] A. Floer, Trans. Am. Math. Soc. 321, 647 (1990).

[21] H.B. Keller, in Numerical Solution of Bifurcation and Nonlinear Eigenvalue Problems, edited by P. Rabinowitz, Application of Bifurcation Theory Vol. 359 (Academic Press, New York, 1977).

[22] C. Froeschlé, M. Guzzo, and E. Lega, Science 289, 2108 (2000).

[23] M. Guzzo, E. Lega, and C. Froeschlé, Physica (Amsterdam) 238D, 1797 (2009).

[24] E. P. Wigner, Trans. Faraday Soc. 34, 29 (1938). 